doi: 10.2306/scienceasia1513-1874.2012.38.268

\title{
Efficacy evaluation of the fibroblast-seeded collagen/chitosan scaffold on application in skin tissue engineering
}

\author{
Sirintip Intaraprasit ${ }^{\mathrm{a}}$, Atchariya Faikrua ${ }^{\mathrm{b}}$, Anuphan Sittichokechaiwut ${ }^{\mathrm{c}}$, Jarupa Viyoch $^{\mathrm{a}, *}$ \\ a Department of Pharmaceutical Technology, \\ Faculty of Pharmaceutical Sciences and Centre of Excellence for Innovation in Chemistry, \\ Naresuan University, Phitsanulok 65000 Thailand \\ b School of Medical Sciences, University of Phayao, Phayao 56000 Thailand \\ c Department of Preventive Dentistry, Faculty of Dentistry, Naresuan University, Phitsanulok 65000 Thailand
}

*Corresponding author, e-mail: jarupav@nu.ac.th, jarupaviyoch4@yahoo.com

Received 9 Nov 2011

Accepted 11 Sep 2012

\begin{abstract}
There remains a need for dressings which aid the healing of chronically refractory wounds. To this end, we formulated and tested a collagen/chitosan scaffold supporting basic fibroblast growth factor (bFGF) producing cells. Collagen was blended with chitosan derived from crab shell (in ratio 7:3 of 3\% weight of total polymer), crosslinked with glutaraldehyde, and then cast into a film. Tensile strength and elongation at break values of the scaffold was $8.5 \pm 0.1 \mathrm{MPa}$ and $2.4 \pm 0.6 \%$, respectively. The swelling degree was $77 \pm 4 \%$, and the weight remaining after collagenase degradation for 1 month was $30.5 \pm 6.3 \%$. Our scaffolds showed bio-compatibility because they encouraged attachment and proliferation of spindle-shaped human dermal fibroblasts (HDFs) which secreted surface-bound bFGF. Next, the fibroblast-seeded scaffold was prepared by seeding HDFs on the collagen/chitosan scaffold $\left(5 \times 10^{5}\right.$ cells $\left./ \mathrm{cm}^{2}\right)$ and further cultured for 7 days. These fibroblast-seeded scaffolds were used to dress full depth wounds in domestic pigs. The wounds with fibroblastseeded scaffolds showed reduced swelling at day 7 and at all time points (7, 14, and 21 days), and re-epithelialization was faster than controls. Thus at day 21 of treatment, wounds treated with the fibroblast-seeded scaffold provided the highest percent re-epithelialization $(69.7 \pm 9.6 \%)$, which was higher than untreated $(49.9 \pm 6.3 \%)$ and cell-free scaffold treatment $(54.3 \pm 6.9 \%)$. The wound tissue of the fibroblast-seeded scaffold treated group showed regular arrangement of host collagens and the rete ridge-like structure at the epidermal layer was also found at day 21 . The results indicate the potential of the fibroblast-seeded scaffold for application in skin tissue engineering.
\end{abstract}

KEYWORDS: wound healing, basic-fibroblast growth factor, re-epithelialization

\section{INTRODUCTION}

Skin loss is a critical human health problem and surgical reconstruction still leaves scope for improvement. Patients who receive extensive surface and deep wounds require rapid healing to prevent dehydration and infection. Exogenous administration of growth factors, such as basic fibroblast growth factor (bFGF), through a suitable delivery system is one approach to accelerate healing of acute and chronic wounds ${ }^{1-5}$. However, this approach is limited by the susceptibility to degradation and short half-life of growth factors dispersed or encapsulated in the delivery system, resulting in low success rates for cutaneous repair. Skin tissue engineering is another approach to resolve such limitations. In essence, it uses scaffold material that allows fibroblasts, the main component of dermal cells, to grow and function in a controlled environ- ment. After the fibroblasts are cultured on such scaffold for an optimal period, the cell-seeded scaffold is transplanted into the wound area. The presence of growth factors and cytokines secreted by these living fibroblasts, together with a biocompatible scaffold, promotes migration, adhesion, and proliferation of host skin cells, thus boosting the rate of cutaneous restoration.

Several biocompatible and biodegradable polymers such as poly(glycolic acid), poly(lactic acid), collagen, gelatin, cellulose, hyaluronate, chitosan, and alginate have been explored as scaffolds for tissue engineering applications ${ }^{6}$. Among materials used, the naturally derived polymers including collagen ${ }^{7-9}$ and chitosan ${ }^{10-12}$ have received special attention because of their biological and chemical similarities with natural tissues. Collagen dressings have been used to address a variety of surgical problems including 
burns and wounds. However, its fast degradation rate and low mechanical strength pose limitations in tissue engineering. Chitosan, a biocompatible polymer, is an attractive alternative because it induces local cell proliferation and it is stable enough to be integrated with the host tissue ${ }^{13}$. Moreover, experimental open wounds in dogs and cats treated with chitosan do not leave a wide scar after healing ${ }^{14}$. Nevertheless, while chitosan is not cytotoxic, it may inhibit fibroblast proliferation $^{15}$. As such, chitosan is unsuitable for in vitro fibroblast cultivation but still suggested its use in combination with other materials ${ }^{16}$. By blending both collagen and chitosan, there is the prospect that the resultant scaffold may overcome the limitations of each polymer.

The present study, therefore, aimed to demonstrate that a fibroblast-seeded collagen/chitosan scaffold is an efficacious skin tissue engineering material. This is based on the expectation that these composite scaffolds produce growth factors thus further promoting wound healing compared to cell-free scaffolds. To do this, we sought to: create a more robust and biocompatible scaffold suited to rigours of the clinical setting, show that human fibroblasts could proliferate and grow on these scaffolds and produce bFGF protein in a bioavailable form, and show that the fibroblastseeded scaffolds would promote healing using an experimental animal where the wound closure is similar to that of humans (the pig). The results show that all three objectives were met; in particular, full depth skin wounds showed superior healing rates without leaving a scar.

\section{MATERIALS AND METHODS}

\section{Materials}

Type I collagen was isolated from bovine tendon, according to our previous study ${ }^{17}$. Chitosan used in the present study was derived from crab shell (crab chitosan, molecular weight of 100000-1000000 and more than $90 \%$ degree of deacetylation, Bannawach Bio-line Co. Ltd., Chonburi, Thailand). Our previous study indicated the good mechanical properties of the crab chitosan over chitosan derived from shrimp shell or squid pen ${ }^{18}$. Glutaraldehyde (GA) was purchased from Fluka Chemie $\mathrm{GmbH}$, Buchs, Switzerland. Dulbecco's Modified Eagle Medium (DMEM) was purchased from Sigma-Aldrich Co., Missouri, USA. Foetal bovine serum (FBS) was purchased from Invitrogen, California, USA. Human dermal fibroblast (HDF, Lot No. C12366) was purchased from Promocell, Eppelheim, Germany, and 2,3-bis(2-methoxy4-nitro-5-sulfophenyl)-5-[(phenylamino)-carbonyl]-
2H-tetrazolium hydroxide (XTT) was purchased from Boehringer Mannheim, Mannheim, Germany. Rabbit polyclonal antibody against bFGF (ab58618) and goat polyclonal against rabbit IgG-H\&L [FITC] (ab6717) were purchased from Abcamplc., Cambridge, UK.

\section{Preparation and characterization of the collagen/chitosan scaffolds}

A simple casting technique was used to prepare the scaffolds. The total amount of polymer used was fixed at $3 \% \mathrm{w} / \mathrm{v}$ and the proportion of collagen to chitosan was $7: 3^{17}$. Type I collagen and chitosan were separately dissolved in $0.5 \mathrm{M}$ acetic acid solution. The collagen and chitosan solutions were mixed for $1 \mathrm{~h}$ using a magnetic stirrer and GA $(0.1 \%$ of the total polymer weight) was added. The casting solutions were further stirred at room temperature for $1 \mathrm{~h}$ before pouring into a clear dried glass Petri dish in a dustfree atmosphere. After drying at room temperature, the resultant scaffold films were peeled off the glass Petri dish, vacuum sealed in a plastic bag, and stored at room temperature. All these films had a thickness of $60 \pm 10 \mu \mathrm{m}$ as verified by using Micrometer (Mitutoyo, Kanagawa, Japan).

Scanning electron microscope (SEM, 1455VP, Leo Electron Microscopy, Inc, Cambridge, UK) was used to observe the surface morphology of the scaffold films and a tensometer (Instron 8872, Instron Ltd., Buckinghamshire, UK) was used to determine their mechanical properties. To measure swelling, a circular scaffold was cut-out, weighed $\left(w_{0}\right)$, placed into distilled water at room temperature for $24 \mathrm{~h}$, blotted, and reweighed $(w)$. Its swelling ratio was defined as the ratio of weight increase $\left(w-w_{0}\right)$ to the initial weight ${ }^{9}$. To determine its enzymatic degradation, the scaffold film was incubated in collagenase $(200 \mathrm{U} / 5 \mathrm{~g}$ of collagen) in $10 \mathrm{ml}$ phosphate buffered saline (PBS, $\mathrm{pH} 7.4$ ) at $37^{\circ} \mathrm{C}$ for 1 month ${ }^{17,19}$ and the enzyme was refreshed every 2 days. The percentage of remaining weight was calculated from $w / w_{0}$. Each value was averaged from three measurements.

Infrared spectra (wavenumber 4000-600 $\mathrm{cm}^{-1}$ ) of GA, the films of collagen, crab chitosan, and GAcrosslinked collagen/chitosan were recorded by FTIR spectrometry (Model GX series, Perkin Elmer, Connecticut, USA).

\section{Preparation of the fibroblast-seeded collagen/chitosan scaffolds}

The scaffold acidity was neutralized by soaking in $10 \% \mathrm{NH}_{4} \mathrm{OH}$ and the scaffolds were rinsed with sterilized distilled water 3 times. Then they were immersed in $75 \%$ ethanol for $24 \mathrm{~h}$ for sterilization and rinsed 
with sterilized PBS for 3-4 times ${ }^{20}$. Scaffold films were equilibrated for another $24 \mathrm{~h}$ period in the culture medium (DMEM supplemented with 10\% FBS) before use. These treated scaffolds were placed into tissue culture plates and HDFs were seeded on them at $7.5 \times 10^{5}$ cells $/ \mathrm{cm}^{2}$ and incubated at $37^{\circ} \mathrm{C}$ in $5 \% \mathrm{CO}_{2}$ for 7 days. The culture medium was changed every 3 days. The cells could not penetrate the scaffold but grew over the surface to $\sim 90 \%$ confluence.

\section{Determination of in vitro biocompatibility types of behaviour of the collagen/chitosan scaffolds}

Determination of cell adhesion and proliferation: After 1, 3, 24, 48, 72, 120, and $144 \mathrm{~h}$ (7 days) of cultivation, the fibroblast-seeded scaffolds were rinsed with sterilized PBS to remove non-adherent cells and they were placed into new wells. The number of cells remaining on the scaffolds was quantified by XTT assay as used previously ${ }^{17,21}$. Briefly, the required amount of serum-free DMEM and XTT labelling mixture was added to wells containing the cell-adherent scaffolds. After incubation for $4 \mathrm{~h}$ at $37{ }^{\circ} \mathrm{C}$ under $5 \% \mathrm{CO}_{2}$, the absorbance of the supernatant was measured at $490 \mathrm{~nm}$ by spectrophotometry (Model CeresUV900C, Bio-tek Instrument, Winooski, Vermont, USA). The study was run in triplicate. For separate scaffolds, the morphology of cells attached on the scaffold was observed under SEM. Cell distribution over the scaffold was assessed by MTT staining and light microscopy.

bFGF expression in fibroblasts cultured on the scaffolds: Basic-fibroblast growth factor (bFGF) produced by fibroblasts has an important role in wound healing. Therefore, its expression in fibroblasts was used to indicate their relevant function whilst cultured in the scaffold films.

After culturing for 7 days, the cell-seeded scaffolds were washed with PBS and fixed with ice-cold methanol for $15 \mathrm{~min}$ at room temperature. The fixed samples were washed with ice-cold PBS, permeabilized by incubating for $10 \mathrm{~min}$ in PBS containing $0.1 \%$ Triton-X 100 at room temperature, and washed with PBS 3 times for 5 min. Non-specific antibody binding was blocked with 5\% bovine serum albumin (BSA) in PBS/Triton for 30 min. The samples were incubated with monoclonal against rabbit polyclonal to bFGF (diluted in 1\% BSA/PBS/Triton) in humidified chamber for overnight at $4{ }^{\circ} \mathrm{C}$. After washing 2 times with PBS/Tritonand once with PBS, 5 min each, the samples were further reacted with goat anti-rabbit polyclonal [FITC] (diluted appropriately in PBS/Triton/1\% BSA; 1:500) for $1 \mathrm{~h}$ at room temper- ature in the dark. Afterwards, samples were washed 3 times for 5 min each with PBS/Tritonand once for 5 min with PBS. The samples were viewed under a fluorescence microscope (Axio Observer.A1, Carl Zeiss, Oberkochen, Germany).

\section{Efficiency of the fibroblast-seeded scaffolds on wound healing in domestic pigs}

Domestic pigs (2-3 months old, weighing $\sim 20 \mathrm{~kg}$ ) were used to assess the efficacy of the fibroblastseeded collagen/chitosan scaffolds on healing of full thickness surgical wounds. The protocol was approved by the Ethical Committee of the Animal Research of Naresuan University, Phitsanulok, Thailand. All surgical procedures were carried out under proper anaesthesia and pain relief. Full thickness surgical wounds were created by skin removal $(10 \mathrm{~mm} \times 10 \mathrm{~mm}$, depth of $2.5 \pm 0.2 \mathrm{~mm})$ along each side of dorsal midline area of 3 pigs (6- 8 wounds in each pig). After that, wounds were dressed with collagen/chitosanscaffold film or those which had been fibroblast-seeded (pre-cultured for 7 days as procedure mentioned above, cell-seeded side opposing the wound). Elastic gauze was taped around the animal to cover on the wounds.

For each animal, the treatments were divided into 3 categories; non-treatment wound, wound treated with the fibroblast-seeded collagen/chitosan scaffold, and wound treated with the cell-free collagen/chitosan scaffold.

At 7, 14, and 21 days, the animals were reanaesthetised. The individual wound sizes were measured and recorded by photography and for each animal 2 wounds were dissected free, and the crater site redressed. Percent re-epithelialization was calculated from the size of wound as ${ }^{22}\left(S_{0}-S\right) / S_{0}$, where $S_{0}$ was wound size at day 0 and $S$ was wound size at either day 7, 14, or 21 .

Wound tissues were also taken for histology evaluation. The samples were soaked in $10 \%$ buffered neutral formalin solution for $12 \mathrm{~h}$ at room temperature and embedded in paraffin at less than $56^{\circ} \mathrm{C}$. The blocks were cut $5 \mu \mathrm{m}$ and floated on to warm water $\left(45^{\circ} \mathrm{C}\right)$ containing $2 \%$ gelatin to prevent peeling off of the sample. The sections were deparafinized, rehydrated, and stained with haematoxylin and eosin.

\section{Statistical analysis}

All data were expressed as the mean \pm standard deviation. Student's $t$-test was used for comparison between the averages of two independent groups and ANOVA was used for multiple comparisons. The 
$p$-value of less than 0.05 was considered statistically significant.

\section{RESULTS}

\section{Properties of the collagen/chitosan scaffolds}

In our previous study ${ }^{17}$, we used type I collagen for testing potential scaffolds for wound healing. The scaffolds were intended as temporary dressings to facilitate wound repair because of its abundance in skin tissue. This was blended with crab chitosan because it had superior mechanical properties compared to that derived from shrimp shell or squid pen ${ }^{18}$. However, scaffolds consisting of a high ratio of collagen/crab chitosan (9:1) produced rather weak and brittle scaffolds which were difficult to remove from the glass Petri dish.

Here, the increased proportion of chitosan together with crosslinking with GA strengthened the scaffold (data not shown). Although the scaffold had a gross film sheet-like structure, SEM visualization revealed a cellular structure (Fig. 1). Tensile strength and elongation at break values of the scaffold was $8.5 \pm 0.1 \mathrm{MPa}$ and $2.4 \pm 0.6 \%$, respectively, and the degree of swelling was $76.9 \pm 3.6 \%$.

In the skin, there is continuous remodelling of the connective tissue involving fibroblast proteolytic enzymes and we wanted to verify that the scaffolds would remain intact through the healing period. To test this, the scaffolds were incubated in collagenase for 1 month and, after this, the remaining weight in average was $30.5 \pm 6.3 \%$.

Fig. 2 shows the chemical characteristic of GA, a crab chitosan film, a collagen film, and GA crosslinked collagen/chitosan film. Focusing on the GA crosslinked collagen/chitosan film, the absorption band in the region of $3500-3400 \mathrm{~cm}^{-1}$ represented the amino group, but it was masked by the broad absorption band from the $-\mathrm{OH}$ group. It also showed an imine bond signal at $1655 \mathrm{~cm}^{-1}(\mathrm{C}=\mathrm{N}$ stretching $)$, and peaks at 2850 and $2751 \mathrm{~cm}^{-1}$ indicating an aldehyde $\mathrm{I}(\mathrm{C}-\mathrm{H}$ aldehyde stretching), and a peak at $1714 \mathrm{~cm}^{-1}$ that would indicate an aldehyde II $(\mathrm{C}=\mathrm{O}$ aldehyde stretching) did not appear.

\section{In vitro biocompatibility behaviour of the collagen/chitosan scaffolds}

In the present study, the XTT assay was used to compare the viabilities of fibroblasts growing on the scaffold and tissue culture plate. The data are presented as averaged absorbance values which is proportional to the number of viable cells. As shown in Fig. 3, there was no difference between these two cell substrates

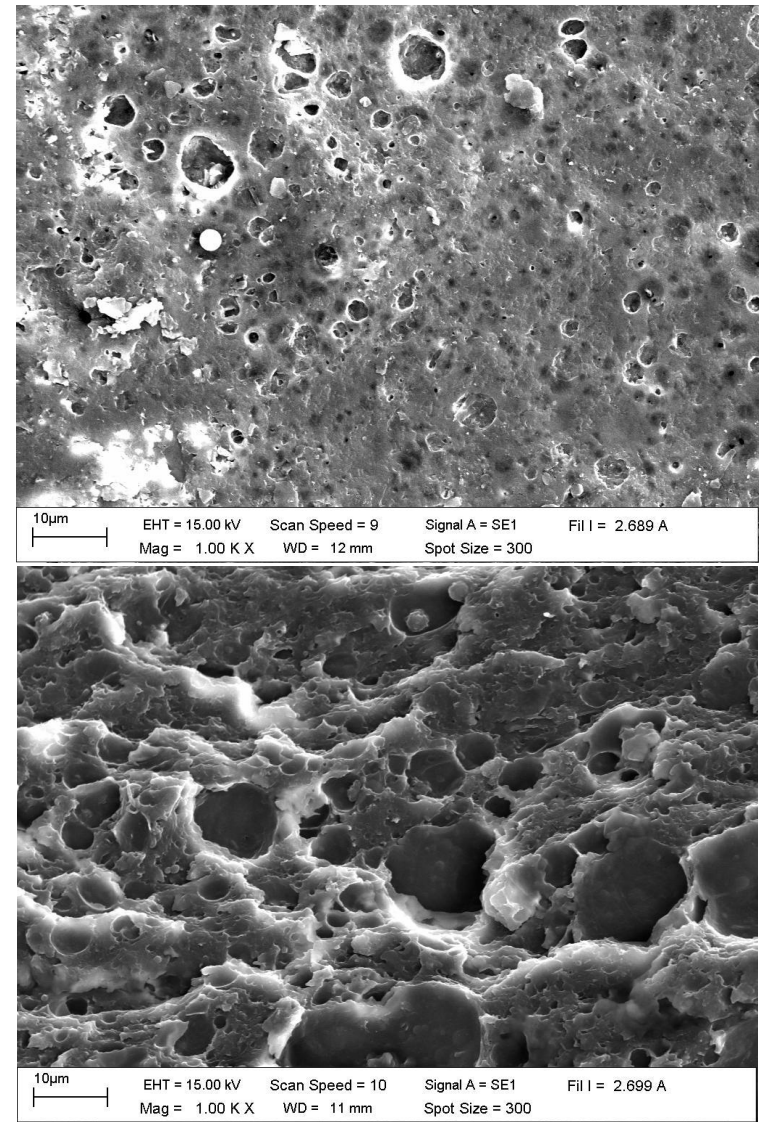

Fig. 1 SEM photographs indicating surface morphology of a collagen/chitosan scaffold at $1000 \times$; upper panel: top view and lower panel: a cross sectional view.

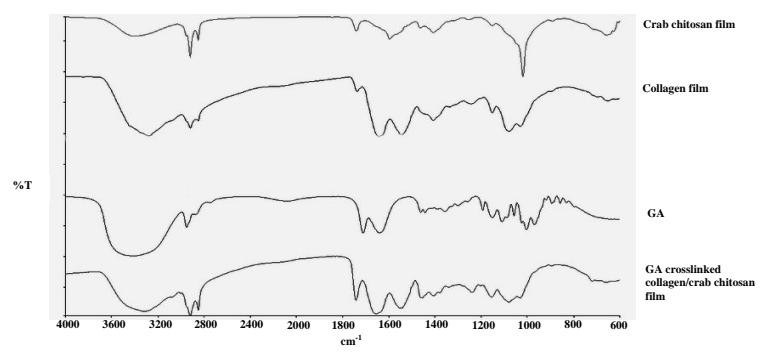

Fig. 2 Wide scan FT-IR spectra for GA and films of crab chitosan, collagen, and GA crosslinked collagen/chitosan.

after cultivation for 7 days.

Scaffolds populated with fibroblasts are shown in Fig. 4. Cells were spindle-shaped and adhered well on the rough surface of the scaffold. Cross sections of cell-seeded scaffold showed no migration of cells into scaffold matrix (data not shown). Viable cells on the scaffold surface were stained with MTT. These HDF cells were evenly distributed (i.e., were not 


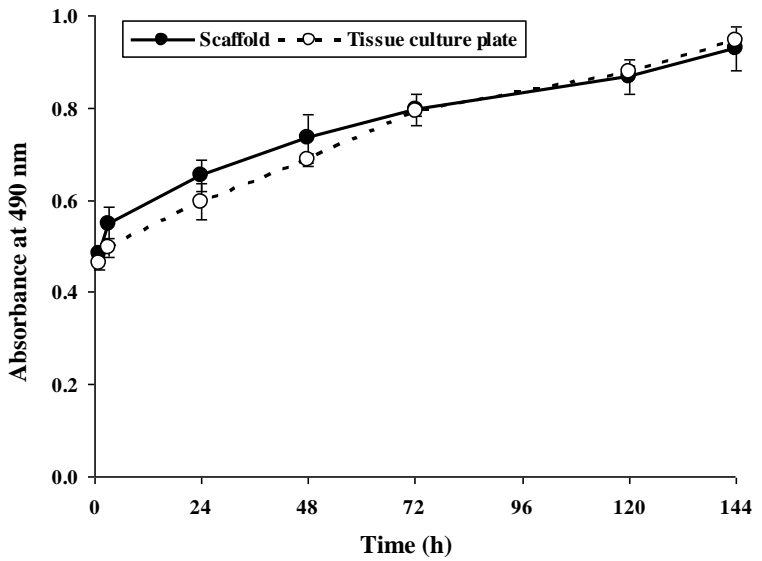

Fig. 3 Proliferation of HDFs on the scaffold and tissue culture plate measured by XTT assay. Data are present as mean \pm SD of absorbance at $490 \mathrm{~nm}(n=3)$.

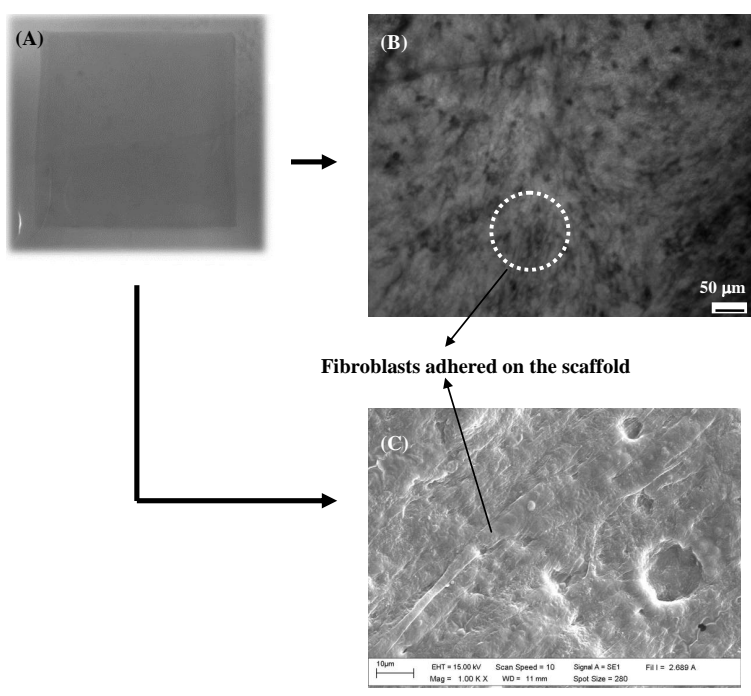

Fig. 4 (A) Characteristic of the fibroblast-seeded collagen/chitosan scaffold immersed in DMEM, and distribution and morphology of HDFs cultured on the scaffold for 7 days; (B) cells stained with MTT indicating distribution pattern of viable cells on the scaffold and (C) SEM photographs at $1000 \times$ indicating morphology of cells adhered on the scaffold.

clumped together) and were near confluence as shown in Fig. 4B. There appeared to be strong expression of bFGF which was confined to the plasma membranes of fibroblasts (see arrows in Fig. 5). This was irrespective of whether cells were cultured on scaffolds or on tissue culture plates.
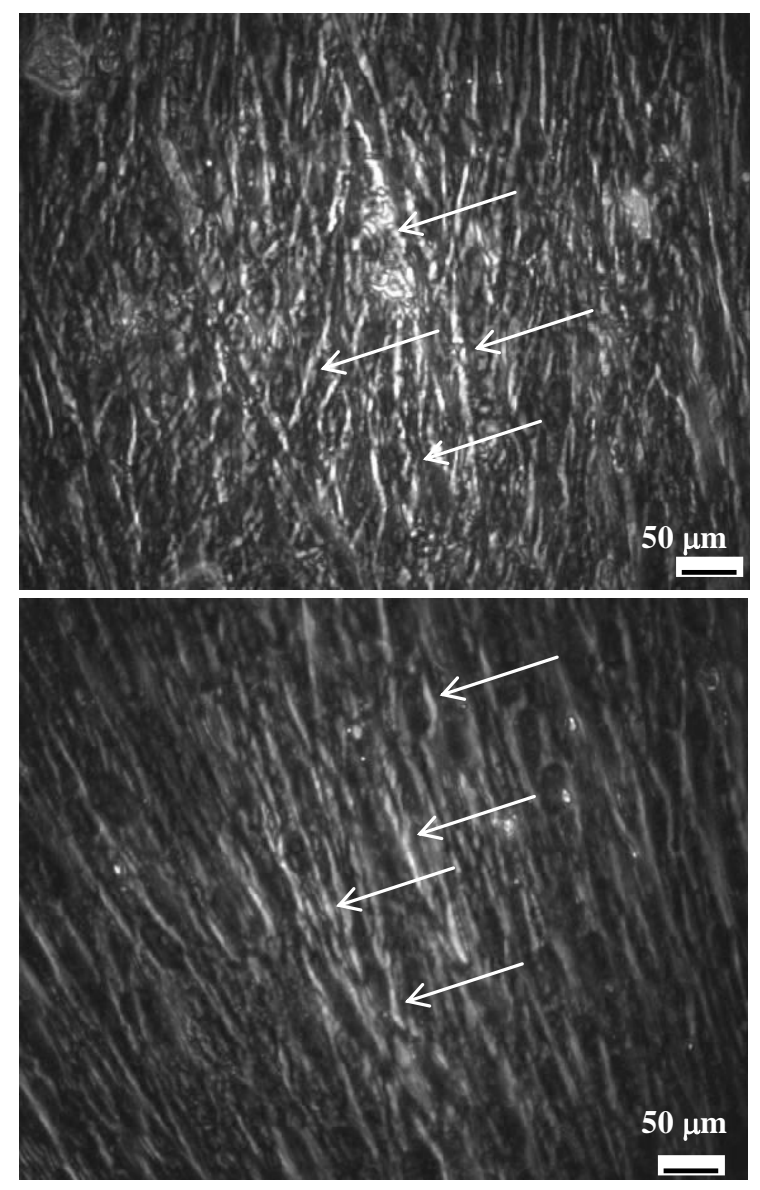

Fig. 5 Immunofluorescent photographs for bFGF of HDFs cultured on the scaffold (upper panel) and the tissue culture plate (lower panel) for 7 days. The arrows indicate examples of bFGF-bound cell surface.

\section{Fibroblast-seeded scaffolds accelerate wound healing in pigs}

The efficiency of the cell-seeded collagen/chitosan scaffolds on the healing of full thickness surgical wounds was tested in domestic pigs and compared to cell-free scaffold. The scaffolds proved durable and flexible enough as wound dressings without fragmentation. During healing, wound photographs of all treatments were recorded (Fig. 6). At day 7, none of the wounds were closed but covered in dark red scabs. However, both the non-treated and cell-free scaffold dressed-wounds showed considerable swelling and the scabs were ruptured. In contrast, the wounds dressed with the fibroblast-seeded scaffolds showed no obvious swelling and retained intact scabs. None of the wounds produced pus which otherwise would have indicated infection. 


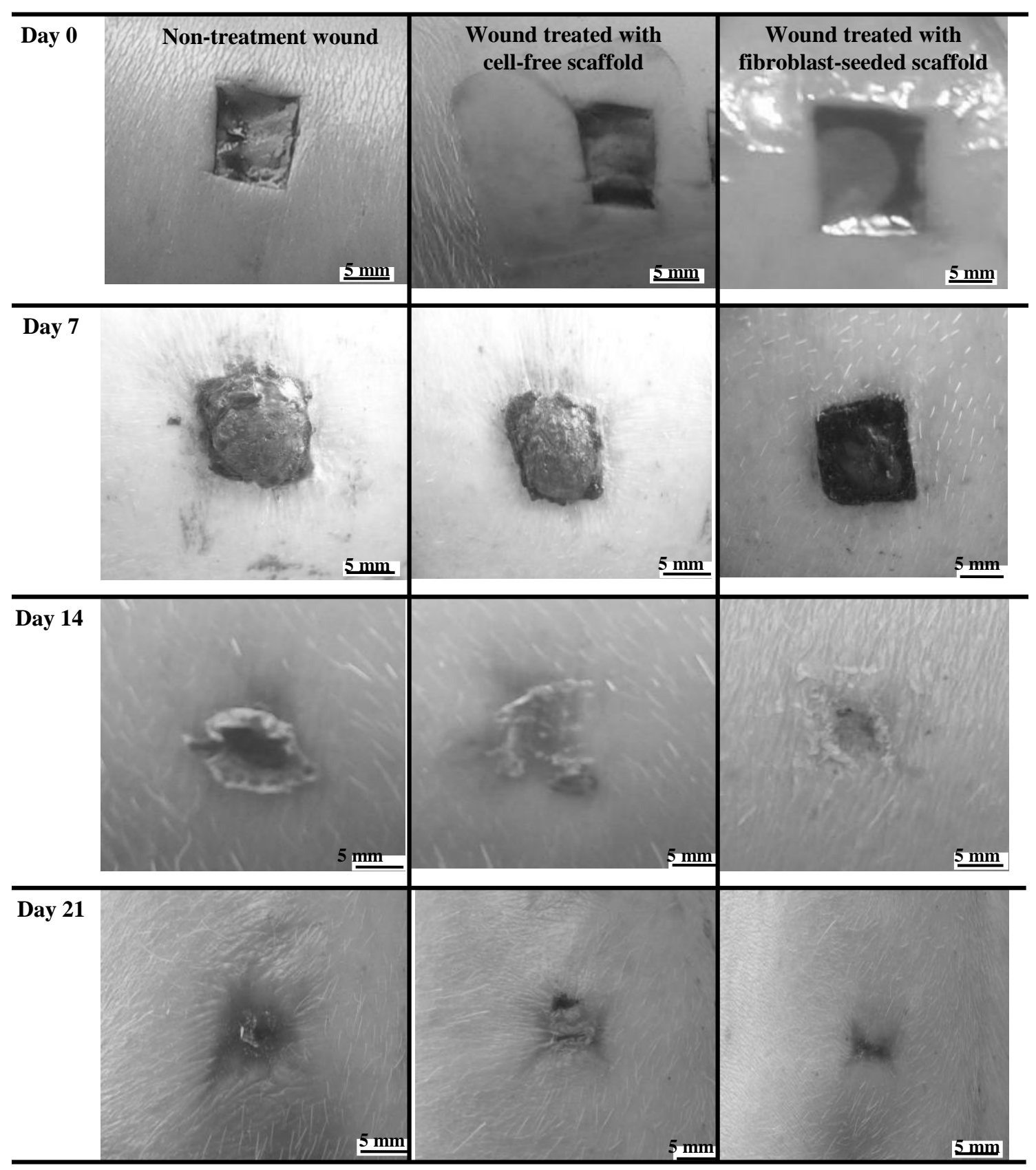

Fig. 6 Appearance of wounds after treated with different conditions for 7, 14, and 21 days.

At day 14, fragments of the scab became detached and the swellings had subsided. While the undressed wounds still showed an extensive wound crater, the wounds treated with fibroblast-seeded scaffold were much reduced in extent and showed cleaner craters.

At 21 days, the untreated wounds were still extensive and no longer square while the cell-seeded wounds had almost completely closed over.
The degree of re-epithelialization is shown in Fig. 7. The untreated and un-seeded scaffold-dressed wounds showed apparent enlargement of the crater area which was due to wound swelling and drying of skin around the crater rim. Meanwhile, the wounds dressed with fibroblast-seeded scaffolds showed no such adverse effects confirming the macroscopic observations (Fig. 6). However, at 14 and 21 days, all the 


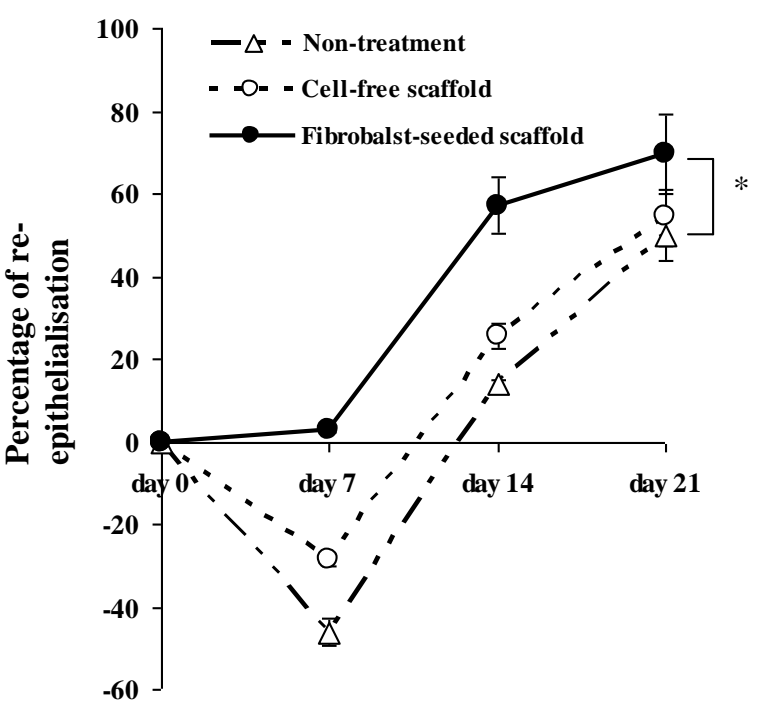

Fig. 7 Time-course for re-epithelialization after wounding and treatments as in Fig. 6. Statistically significant from non-treatment $(n=3), * P<0.05$ (ANOVA).

wounds showed some progress in re-epithelialization but those dressed with cell-seeded scaffolds showed far greater advancement. Eventually, after 8 weeks, all treated areas showed no sign of residual scarring.

Fig. 8 shows the histology of an unwound skin showing the characteristic structure with a thinepidermal layer and randomly distributed collagen fibres. At day 7 of treatment, all groups showed thick epidermal layers. Interestingly, there was a suggestion of dermal formation in the fibroblast-seeded scaffold group. After 21 days, the thick epidermis was still present in the non-treated group whereas thinner epidermal layers were found in both the cellfree and fibroblast-seeded scaffold wounds. Wounds treated with the fibroblast-seeded scaffolds showed regularized collagen and the rete ridge-like structure at the epidermis layer was also becoming apparent.

\section{DISCUSSION}

Scaffolds of collagen/crab chitosan crosslinked with GA were cellular structures which encouraged cell adhesion ${ }^{23}$. Its maximum tensile strength $(8.5 \pm 0.1 \mathrm{MPa})$ lies within the range of $8-10 \mathrm{MPa}$ previously recommended ${ }^{18,24}$, although the elongation tolerated $(2.4 \pm 0.6 \%)$ was lower than reported previously ${ }^{25,26}$. Additionally, the scaffolds were easy to handle and resisted rupture during implantation.

The hydrophilic nature imparts swelling due to medium uptake and if excessive risks change in shape or rupture of the scaffold. However, our scaffolds swelled by $77 \%$ without compromising shape and strength and are well within the recommended 80fold $^{27}$. Collagenase digestion of scaffolds for 1 month removed $\sim 70 \%$ of the dry mass suggesting that these films would be functional within an acute healing $\operatorname{period}^{27}$.

FT-IR analysis of scaffolds showed that imine bonds were present as formed by covalent bonding of the GA $-\mathrm{COH}$ with amino groups $\left(-\mathrm{NH}_{2}\right)$ of collagen and/or chitosan ${ }^{28,29}$. The $\mathrm{GA}-\mathrm{COH}$ peaks were absent from crosslinked scaffolds implying that all these potentially cytotoxic moieties had been reacted.

The scaffolds were clearly biocompatible in vitro, since HDFs adhered well to the rough surface and proliferated into the characteristic spindle-shape of fibroblasts. Thus our scaffolds are suited to tissue engineering. Various cell-substrate interactions contribute to adhesion. These may include electrostatic attraction to unreacted amino groups and integrin binding to collagen ${ }^{30-32}$. HDFs grown on scaffolds were evenly distributed without clustering which is critical for successful tissue engineering ${ }^{33}$.

It is known that bFGF promotes wound granulation, fibroblast proliferation and migration ${ }^{34-37}$, and proliferation of keratinocytes ${ }^{38-40}$ thereby stimulating re-epithelialization. bFGF is translocated in an isoform selective manner to the nucleus, cytosol, or exported to the outer surface ${ }^{41-43}$ where it complexes with heparin to form a reservoir of active growth factor ${ }^{44}$. Our immunohistochemistry shows that surface-bound bFGF was the predominant form in cells cultured on plastic and scaffolds. This reaffirms the scaffold biocompatibility and shows that bFGF protein is expressed in a form available for humoural signalling ${ }^{44}$.

To test our scaffold performance in wound healing, we opted for pigs because their skin has morphological and functional similarities to human skin: epidermal and dermal thicknesses, sparsity of hair, and the presence of subcutaneous fat. Importantly, healing is dominated by granulation and epithelialization $^{45}$ whereas rodents rely on wound contraction ${ }^{46}$. Wounds dressed with our HDF-seeded scaffolds were noteworthy by their cleaner appearance and reduced swelling compared to controls. Another critical stage is re-epithelialization and this was substantially more advanced than the controls, which is likely the result of bFGF stimulation of keratinocyte hyperplasia ${ }^{38,39}$.

According to the histology study, we found that all groups showed thick epidermal layer at day 7 of treatment. This is because keratinocytes in epidermis started to migrate and proliferate for the recovery of the wound. Interestingly, the formation of dermis found in the fibroblast-seeded scaffold treated group 

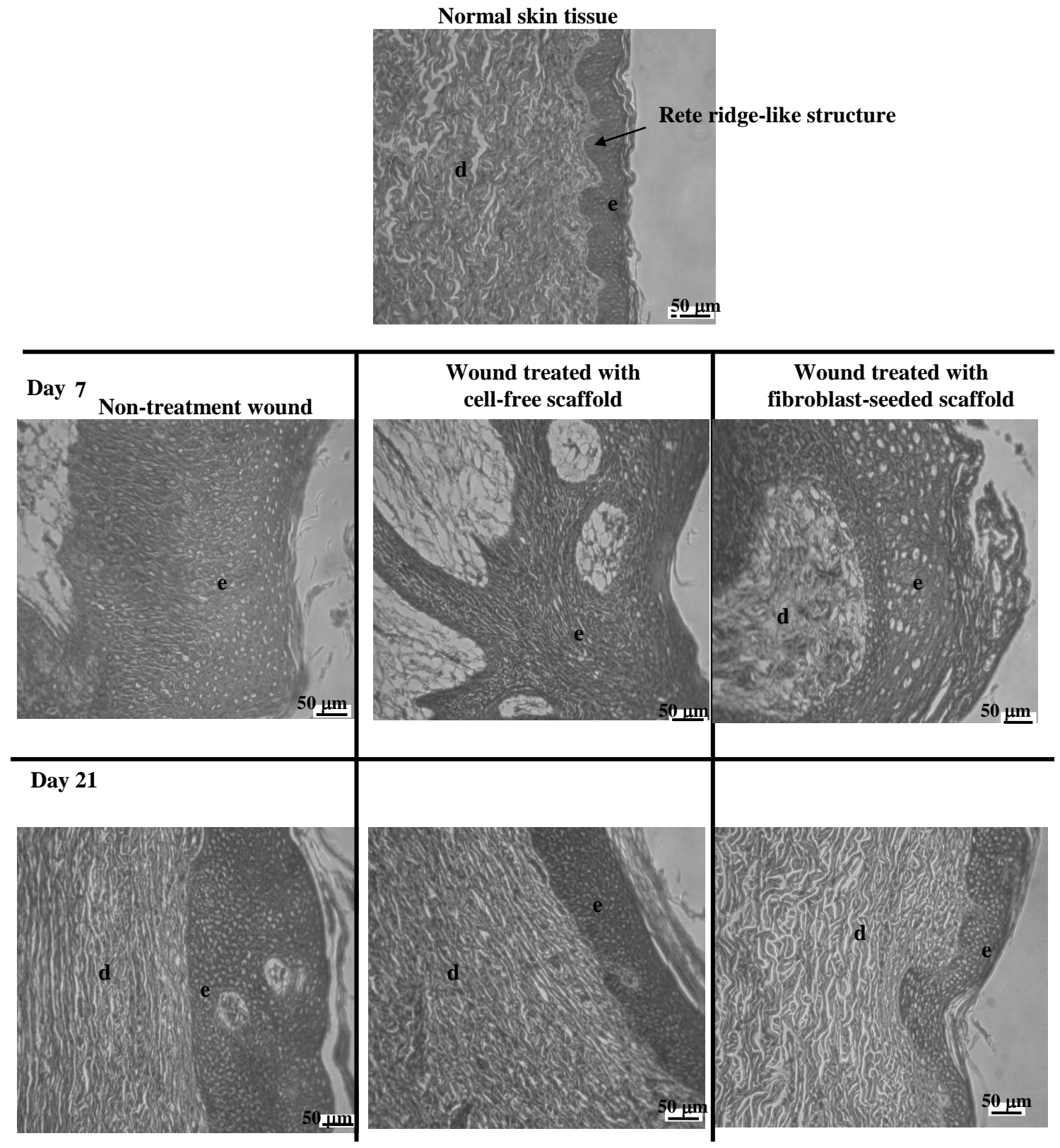

Fig. 8 Histological photographs at $20 \times$ of wound treated with different conditions for 7 and 21 days; e = epidermis layer, $\mathrm{d}=$ dermis layer.

indicated that fibroblasts started to migrate in wound which is one characteristic of proliferation phase ${ }^{47}$. According to the in vitro study, HDFs did not migrate into the prepared scaffold. Therefore, the formed dermis might have been generated by host cells initially receiving active growth factors produced from the seeded HDFs. A previous study reported that bFGF is well conserved among species and its effects are not species specific ${ }^{48}$.
At day 21 of the treatment, regular formation of collagens at the dermis and the rete ridge-like structure at the epidermis were found in the fibroblast-seeded scaffold treated group. Again, the results indicate that the seeded cells would produce the active growth factors which are necessary for completed wound healing and indicate the potential of the fibroblastseeded collagen/chitosan scaffold for application in wound healing. 
Acknowledgements: This study was supported by the National Research Council of Thailand. We also thank a grant for graduate student from the Centre of Excellence for Innovation in Chemistry (PERCH-CIC), Office of the Higher Education Commission, Thailand. The authors would like to acknowledge the Faculty of Pharmaceutical Sciences, Naresuan University for their support. In addition, we thank Dr Charles Norman Scholfield for his valuable discussions.

\section{REFERENCES}

1. Ishihara M, Obara K, Ishizuka T, Fujita M, Sato M, Masuoka K, Saito Y, Yura H, Matsui T, hattori H, Kikuchi M (2003) Controlled release of fibroblast growth factors and heparin from photocrosslinked chitosan hydrogels and subsequent effect on in vivo vascularization. J Biomed Mater Res 64, 551-9.

2. Cross SE, Roberts MA (1999) Defining a model to predict the distribution of topically applied growth factors and other solutes in excisional full-thickness wounds. J Investig Dermatol 112, 36-41.

3. Fu X, Shen Z, Chen Y, Xie J, Guo Z, Zhang M, Sheng $Z$ (1998) Randomized placebo-controlled trial of use of topical recombinant bovine basic fibroblast growth factor for second-degree burns. Lancet 352, 1661-4.

4. Cini J, Finkenaur A (1998) Gel formulations containing growth factors. US Patent no. 5705485.

5. Dinbergs ID, Brown L, Edelman ER (1996) Cellular response to transforming growth factor- $b 1$ and basic fibroblast growth factor depends on release kinetics and extracellular matrix interactions. J Biol Chem 271, 29822-9.

6. Van Vlierberghe S, Dubruel P, Schacht E (2011) Biopolymer-based hydrogels as scaffolds for tissue engineering applications: A review. Biomacromolecules 12, 1387-408.

7. Badylak SF (2004) Xenogeneic extracellular matrix as a scaffold for tissue reconstruction. Transpl Immunol 12, 367-77.

8. Gomathi K, Gopinath D, Rafiuddin AM, Jayakumar R (2003) Quercetin incorporated collagen matrices for dermal wound healing process in rat. Biomaterials $\mathbf{2 4}$, 2767-72.

9. Ruszczak Z (2003) Effect of collagen matrices on dermal wound healing. Adv Drug Deliv Rev 55, 1595-611.

10. Azad AK, Sermsinthan N, Chandrkrachang S, Stevens WF (2004) Chitosan membrane as a wound-healing dressing: Characterization and clinical application. $J$ Biomed Mater Res 15, 216-22.

11. Mohy Eldin MS, Soliman EA, Hashem AI, Tamer TM (2008) Chitosan modified membranes for wound dressing applications: Preparations, characterization and bio-evaluation. Trends Biomater Artif Organs 22, 158-68.

12. Jayakumar R, Prabaharan M, Sudheesh Kumar PT, Nair SV, Tamura H (2011) Biomaterials based on chitin and chitosan in wound dressing applications. Biotechnol Adv 29, 322-37.

13. Suh JK, Matthew HW (2000) Application of chitosanbased polysaccharide biomaterials in cartilage tissue engineering: A review. Biomaterials 21, 2589-98.

14. Okamoto Y, Minami S, Matsuhashi A, Sashiwa H, Saimoto H, Shigemasa Y, Tanigawa T, Tanaka Y, Tokura S (1993) Application of polymeric $N$-acetyl-Dglucosamine (chitin) to veterinary practice. $J$ Vet Med Sci 55, 743-7.

15. Chatelet C, Damour O, Domard A (2001) Influence of the degree of acetylation on some biological properties of chitosan films. Biomaterials 22, 261-8.

16. Shahabeddin L, Damour O, Berthod F, Rousselle P, Saintigny G, Collombel C (1991) Reconstructed skin from co-cultured human keratinocytes and fibroblasts on a chitosan crosslinked collagen-GAG matrix. J Mater Sci Mater Med 2, 222-6.

17. Faikrua A, Jeenapongsa R, Sila-asna M, Viyoch J (2009) Properties of $\beta$-glycerol phosphate/collagen/ chitosan blend scaffolds for application in skin tissue engineering. Sci Asia 35, 247-54.

18. Ngoenkam J, Faikrua A, Yasothornsrikul S, Viyoch J (2010) Potential of an injectable chitosan/starch/ $\beta$-glycerol phosphate hydrogel for sustaining normal chondrocyte function. Int J Pharm 391, 115-24.

19. Huang Y (2005) In vitro characterization of chitosangelatin scaffolds for tissue engineering. Biomaterials 26, 7616-27.

20. Pieper JS, Oosterhof A, Dijkstra PJ, Veerkamp JH, Van Knpperelt TH (1999) Preparation and characterization of porous crosslinked collagenous matrices containing bioavailable chondroitin sulphate. Biomaterials 20, 847-58.

21. Phetdee M, Polnok A, Viyoch J (2008) Development of chitosan-coated liposomes for sustained delivery of tamarind fruit pulp's extract to the skin. Int J Cosmet Sci 30, 285-95.

22. Bishop SM, Walker M, Rogers AA, Chen WY (2003) Importance of moisture balance at the wound-dressing interface. $J$ Wound Care 12, 125-8.

23. Levy S, Van Dalen M, Agonafer S, Soboyejo WO (2007) Cell/surface interactions and adhesion on bioactive glass 45S5. J Mater Sci Mater Med 18, 89-102.

24. Adekogbe I, Ghanem A (2005) Fabrication and characterization of DTBP-crosslinked chitosan scaffolds forskin tissue engineering. Biomaterials 26, 7241-50.

25. Wang Y, Lin M, Wang D, Hsieh H (2003) Fabrication of a novel porous PGA-chitosan hybrid matrix for tissue engineering. Biomaterials 24, 1047-57.

26. Wang X, Ma J, Wang Y, He B (2001) Structural characterization of phosphorylated chitosan and their applications as effective additives of calcium phosphate cement. Biomaterials 22, 2247-55.

27. Ma L, Gao C, Mao Z, Zhou J, Shen J, Hu X, Han C (2003) Colllagen/chitosan porous scaffolds with improved biostability for skin tissue engineering. Bioma- 
terials 24, 4833-41.

28. Berger J, Reist M, Mayer JM, Felt O, Peppas NA, Gurny R (2004) Structure and interactions in covalently and ionically crosslinked chitosan hydrogels for biomedical application. Eur J Pharm Biopharm 57, 19-34.

29. Monteiro Jr OAC, Airoldi C (1999) Some studies of crosslinking chitosan-glutaraldehyde interaction in a homogeneous system. Int J Biol Macromol 26, 119-28.

30. Pan H, Jiang H, Chen W (2006) Interaction of dermal fibroblasts with electrospun composite polymer scaffolds prepared from dextran and poly lactide- $\mathrm{Co}$ glycolide. Biomaterials 27, 3209-20.

31. Quirk RA, Chen WC, Davies MC, Tendler SJB, Shakesheff KM (2001) Poly(L-lysine)-GRGDS as a biomimetic surface modifier for poly (lactic acid). Biomaterials 22, 865-72.

32. Heino J (2000) The collagen receptor integrins have distinct ligand recognition and signaling functions. $\mathrm{Ma}$ trix Biol 19, 319-23.

33. Mori T, Okumura M, Matsuura M, Ueno K, Tokura S, Okamoto Y, Minami S, Fujinaga T (1997) Effects of chitin and its derivatives on the proliferation and cytokine production of fibroblasts in vitro. Biomaterials 18, 947-51.

34. Angel DE, Morey P, Storer JG, Mwipatayi BP (2008) The great debate over iodine in wound care continues: A review of the literature. Wound Pract Res 16, 6-21.

35. Tsirogianni AK, Moutsopoulos NM, Moutsopoulos HM (2006) Wound healing: Immunological aspects. Injury 37, S5-S12.

36. Oda Y, Kagami H, Ueda M (2004) Accelerating effects of basic fibroblast growth factor on wound healing at rat palatal mucosa. J Oral Maxillofac Surg 62, 73-80.

37. Kouhara H, Hadari YR, Spivak-Kroizman T, Schilling J, Bar-Sagi D, Lax I, Schlessinger J (1997) A lipidanchored Grb2-binding protein that links FGF-receptor activation to the Ras/MAPK signaling pathway. Cell 89, 693-702.

38. Hakvoort T, Altun V, van Zuijlen PP, de Boer WI, van Schadewij WA, van der Kwast TH (2000) Transforming growth factor-beta(1), -beta(2), -beta(3), basic fibroblast growth factor and vascular endothelial growth factor expression in keratinocytes of burn scars. Eur Cytokine Netw 11, 233-9.

39. O'Keefe EJ, Chiu ML, Payne Jr RE (1988) Stimulation of growth of keratinocytes by basic fibroblast growth factor. J Investig Dermatol 90, 767-9.

40. Maas-Szabowski N, Shimotoyodome A, Fusenig NE (1999) Keratinocyte growth regulation in fibroblast cocultures via a double peracrine mechanism. J Cell Sci 112, 1843-53.

41. Florkiewicz RZ, Baird A, Gonzalez A (1991) Multiple forms of bFGF: Differential nuclear and cell surface localization. Growth Factors 4, 265-75.

42. Bugler B, Amalric F, Prats H (1991) Alternative initiation of translation determines cytoplasmic or nuclear localization of basic fibroblast growth factor. Mol Cell

Biol 11, 573-7.

43. Takeuchi R (2004) The effect of basic fibroblast growth factor on cell cycle in human gingival fibroblasts from nifedipine responder and non-responder. J Oral Sci 46, 37-44.

44. Dowd CJ, Cooney CL, Nugent MA (1999) Heparan sulfate mediates bFGF transport through basement membrane by diffusion with rapid reversible binding. J Biol Chem 274, 5236-44.

45. Sullivan TP, Eaglstein WH, Davis SC, Mertz P (2001) The pig as a model for human wound healing. Wound Repair Regen 9, 66-76.

46. Galiano RD, Michaels J, Dobryansky M, Levine JP, Gurtner GC (2004) Quantitative and reproducible murine model of excisional wound healing. Wound Repair Regen 12, 485-92.

47. Enoch S, Leaper DJ (2008) Basic science of wound healing. Surgery 26, 31-7.

48. Hom DB, Unger GM, Pernell KJ, Manivel JC (2005) Improving surgical wound healing with basic fibroblast growth factor after radiation. Laryngoscope 115, 412-22. 